\title{
Brown tumours of the spine presenting with acute urine incontinence
}

\author{
Wei-Yu Chou MD, Yan-Rong Li MD
}

— Cite as: CMAJ 2017 September 5;189:E1116. doi: 10.1503/cmaj.170142

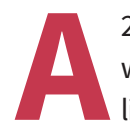

29-year-old man presented to the emergency department with urinary incontinence that began suddenly five days earlier. He had weakness in both legs (muscle power decreased to $4 / 5$ ) with numbness. Upon investigation, the patient had hypercalcemia (serum calcium 3.485 [normal range 1.975-2.475] mmol/L) and hypophosphatemia (serum phosphate 0.581 [normal range 0.775 $1.518] \mathrm{mmol} / \mathrm{L}$ ) with an elevated level of intact parathyroid hormone (1956 [normal range 14-72] ng/mL). Magnetic resonance imaging of his spine showed two lesions over posterior elements of T10 and L4, with compression of the spinal cord at T10 (Figure 1).

The patient had an urgent surgical decompression of the T10 lesion, which, on histology, was composed of round to spindled mononuclear cells and numerous osteoclast-type giant cells with some bony trabeculae. Two weeks after the operation, his neurologic deficits had resolved. Because of pain in his left forearm, the patient had radiography, which showed possible brown tumours (Appendix 1, available at www.cmaj.ca/lookup/suppl/doi:10.1503/ cmaj.170142/-/DC1). A parathyroid scan (Tc-99m sestamibi) and ultrasonography suggested a right parathyroid adenoma, which was confirmed on parathyroidectomy.

Brown tumours are focal reactive osteolytic lesions that occur in hyperparathyroidism. ${ }^{1}$ Multiple brown tumours are more common in the extremities, clavicle, ribs and pelvis than in the spine. ${ }^{2}$ Neurologic deficits caused by spinal brown tumours may be the first evidence of undiagnosed hyperparathyroidism. ${ }^{3}$ Most patients with neurologic deficits are treated initially with urgent decompressive surgery, followed by parathyroidectomy. If treated early, there is satisfactory recovery of neurologic deficits without recurrence. ${ }^{2}$

Brown tumours are often lytic but can also be solid or mixed with fluid content. In patients presenting with these types of lesions on imaging, brown tumours should be considered.

\section{References}

1. Hong WS, Sung MS, Chun KA, et al. Emphasis on the MR imaging findings of brown tumor: a report of five cases. Skeletal Radiol 2011;40:205-13.

2. Khalatbari MR, Moharamzad Y. Brown tumor of the spine in patients with primary hyperparathyroidism. Spine 2014;39:E1073-9.

3. Fargen KM, Lin CS, Jeung JA, et al. Vertebral brown tumors causing neurologic compromise. World Neurosurg 2013;79:208.e1-6.

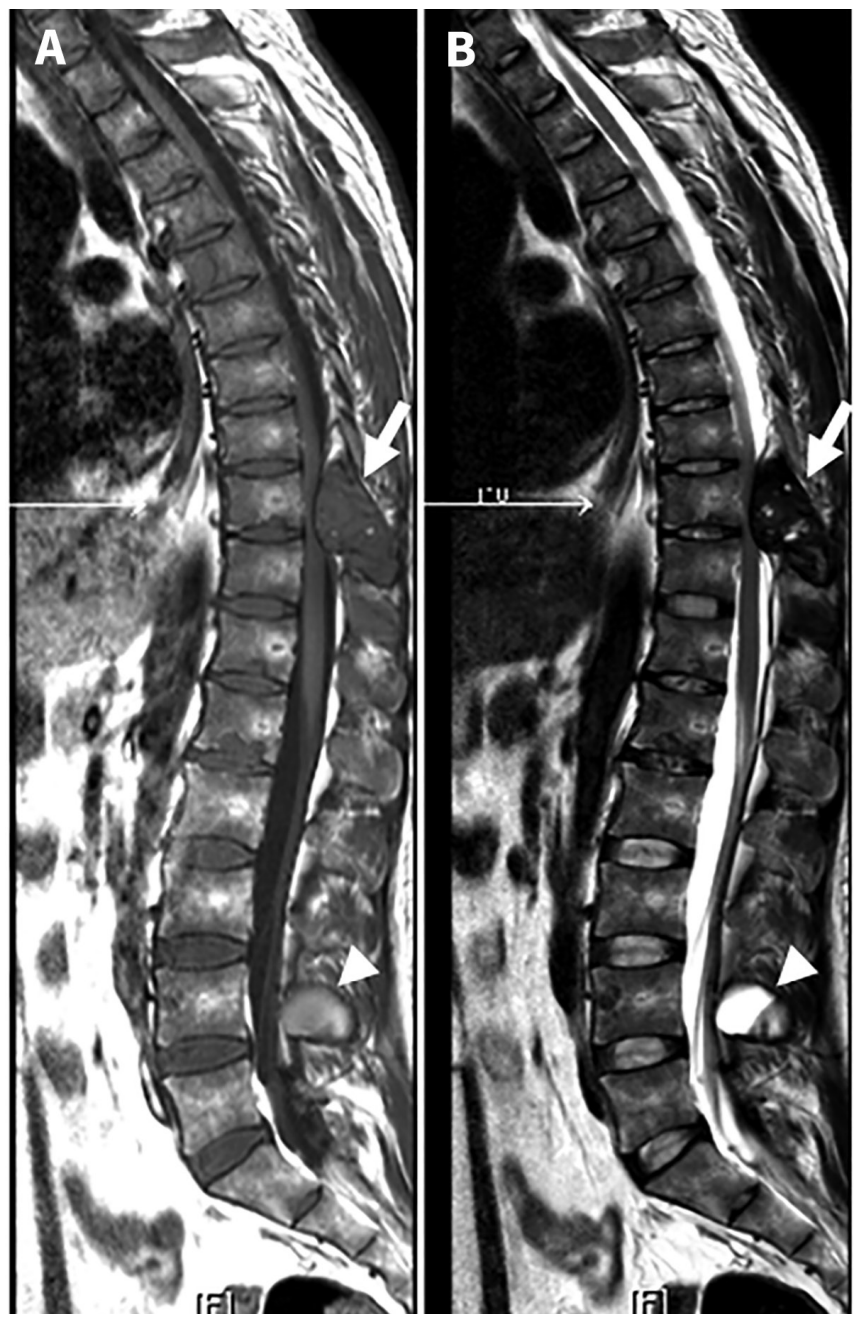

Figure 1: Sagittal (A) $T_{1}$-weighted and (B) $T_{2}$-weighted magnetic resonance images of the spine of a 29-year-old man showing two expansile lesions from posterior elements of T10 and L4. The lesion at T10 (arrow), which had heterogeneous $T_{1}$-weighted isointensity and $T_{2}$-weighted hypointensity mixed with hyperintensity spots, severely compressed the spinal cord. The mixed cystic and solid lesion at L4 (arrowhead) had heterogeneous $T_{1}$-weighted isointesity with the main section having homogeneous $T_{2}$-weighted hyperintensity.

\section{Competing interests: None declared.}

This article has been peer reviewed.

The authors have obtained patient consent.
Affiliation: Department of Internal Medicine, Division of Endocrinology and Metabolism, Chang Gung Memorial Hospital, Taoyuan City, Taiwan.

Correspondence to: Yan-Rong Li, mr8252@cgmh.org.tw 